\title{
ESTADOS UNIDOS EN LA POLÍTICA EXTERIOR COLOMBIANA: ¿ALIADO INCONDICIONAL?'
}

\author{
Roberto González Arana ${ }^{2}$ - Héctor Galeano David ${ }^{3}$ - Luis Fernando Trejos Rosero ${ }^{4}$
}

Universidad del Norte, Colombia - Corporación Universidad de la Costa, CUC, Colombia - Artículo Tipo 1.

Investigación Científica y Tecnológica - Recibido: 2 de Septiembre 2013 - Aceptado: 27 de Abril 2015

doi: http://dx.doi.org/10.17981/econcuc.36.1.2015.23

\section{RESUMEN}

Este artículo analiza la política exterior de Colombia en el periodo de 1950 a 2010 y la influencia de los EEUU en su diseño y aplicación. Muestra como Colombia afianzó su condición de socio estratégico enmarcando su política exterior en el denominado Réspice Polum ${ }^{5}$, apartándose casi por completo de otras opciones en materia económica y política, a pesar de haber formulado el Réspice Similia ${ }^{6}$. Este trabajo es cualitativo, bibliográfico y descriptivo. Se demostró con él, la necesidad del mercado y empréstitos, durante parte del siglo XX y la incapacidad de Colombia de controlar problemas de seguridad interna. Se concluyó que esto ha hecho que en la relación con EEUU, se configure una dependencia casi estructural, y que ha sido el eje sobre el cual ha girado la actividad internacional de Colombia en lo económico, lo político y particularmente en temas de seguridad interna.

\section{Palabras Clave:}

Respice Polum, Respice Similia, Política exterior, Securitización JEL: F35, F50, F52

\section{Si va a referenciar este artículo}

Gonzáles, R., Galeano, H. \& Trejos, L. (2015). Estados Unidos en la política exterior colombiana: ¿aliado incondicional?, Económicas CUC, 36(1), 79-106.

doi: http://dx.doi.org/10.17981/econcuc.36.1.2015.23

\footnotetext{
${ }^{1}$ Este artículo es una reflexión derivada de la investigación "La política exterior colombiana durante el siglo XX. Objetivos, medios y estrategias", desarrollada en el marco del Grupo de Trabajo CLACSO "Movimientos sociales y movimientos revolucionarios en Centroamérica y el Caribe".

${ }^{2}$ PhD. en Historia de la Academia de Ciencias de Rusia. Profesor titular del Departamento de Historia y Ciencias Sociales, Universidad del Norte. Director del Instituto de Estudios latinoamericanos y Caribeños de la misma institución. Coordinador grupo de trabajo CLACSO Miembro del grupo de investigación Memorias del Caribe, Universidad del Norte.rogonzal@uninorte.edu.co

${ }^{3}$ Universidad de la Costa. Docente e investigador del área de las relaciones internacionales. hgaleano1@cuc.edu.co Estudios de Maestría en Resolución de Conflictos y mediación, PhD (c) Ciencias Sociales de la Universidad del Norte

${ }^{4}$ Doctor en Estudios Americanos, mención en Estudios Internacionales (IDEA/USACH). Docente e investigador del Departamento de Ciencia Política y Relaciones Internacionales de la Universidad del Norte. Investigador asociado del Instituto de Estudios latinoamericanos y caribeños, Universidad del Norte. Miembro del Grupo de Investigación "Agenda Internacional", de la Universidad del Norte. Contacto: trejosl@uninorte.edu.co

${ }^{5}$ El lema Respice Polum (mirar hacia la estrella del norte), se convirtió en el eje de la política exterior colombiana. Esta política, buscaba el establecimiento de una relación bilateral profunda en temas comerciales, fue el marco en el que se ha desarrollado un alineamiento histórico de Colombia a los Estados Unidos.

${ }^{6}$ Buscando relacionarse con estados en niveles de desarrollo similares a Colombia.
} 


\section{INTRODUCCIÓN}

Con la finalización de la Segunda Guerra Mundial, el orden global entró en proceso de transformación hacia un nuevo orden mundial que rigió las reglas de juego de la política internacional hasta 1989. El bipolarismo, como se conoció este periodo, conminó a los estados a alinearse con alguno de los dos "jugadores", considerados como superpotencias, es decir, Estados Unidos y la Unión de Repúblicas Soviéticas (URSS).Colombia, no fue la excepción a este proceso de replanteamiento de la "arena" mundial. A partir de esa coyuntura histórica, el país afianza su irrestricta alianza con los Estados Unidos, enmarcando su política exterior en el Réspice Polum (mirar hacia el norte) propuesto por Marco Fidel Suarez en la década del 20.

Con la paulatina construcción del nuevo sistema internacional, se gestaron conflictos locales o regionales, impulsados por la tensión Este-Oeste. No en vano, los Estados Unidos promulgaron doctrinas orientadas a contener ${ }^{7}$ el comunismo expansionista de la URSS, que para el momento tenía como prioridad al este de Europa. En este escenario, se desata el primer conflicto bélico de la segunda posguerra; la guerra de Corea. La guerra en el lejano continente asiático, evidenció la posición en materia de política exterior de Colombia a partir de mediados de siglo, enmarcado en una adhesión total a los Estados Unidos. Por decisión del presidente Laureano Gómez, el ejército colombiano es el único en participar en el conflicto de la península coreana. Según Meléndez (2014), la participación activa colombiana

\footnotetext{
${ }^{7}$ La contención al socialismo creciente en Europa, se convierte en el principal objetivo de la política exterior de los EEUU. Doctrinas como la Truman, Eisenhower y posteriores como la Reagan enfocan sus prioridades en materia de seguridad internacional, en frenar la expansión del socialismo
}

comenzó con el decreto 3230 de 1950 en el que se disponía del envío de la fragata "Almirante Padilla" y con el decreto 3927 de diciembre de 1950 se creó el Batallón de infantería $\mathrm{N}^{\circ} 1$ Colombia, que sería entrenado en Bogotá y los Estados Unidos y después se uniría a una de las compañías norteamericanas en la zona de combate.

\section{FUNDAMENTOS TEÓRICOS}

\section{El Frente Nacional (1958-1974)}

Con la presidencia del liberal Alberto Lleras Camargo (1958), se inició el Frente Nacional (coalición política y electoral entre los partidos tradicionales, liberal y conservador). En ese momento, las puertas de la democracia se cerraron para todos aquellos grupos, partidos o movimientos políticos que no fueran liberales o conservadores. Desde ese año, el estado de sitio y la exclusión de terceras fuerzas políticas serían una constante en la historia de Colombia. Para el gobierno, la única manera de lograr instaurar el imperio de la paz en el escenario internacional, era apoyando irrestrictamente el accionar de los Estados Unidos.

Según Pardo (1987), de esta manera, Colombia, manifestó su abierta disposición a declarar al "comunismo internacional" como enemigo del hemisferio, asumiendo así, los intereses norteamericanos como propios. A nivel doméstico, hay que destacar lo referido al manejo autónomo (ajeno al control civil) que de la seguridad y el orden público, hicieran las Fuerzas Armadas colombianas.Lo que el presidente Lleras buscaba, era mantener a los militares neutrales frente a los partidos tradicionales y evitar la repetición de un Golpe de Estado. De esta manera, desde 1958 hasta 1990 la Doctrina Lleras, fue el único lineamiento político global en materia militar. 
Ante la ausencia, de una clara estrategia política, que condujera el esfuerzo militar del Estado, el anticomunismo contenido en la Doctrina de la Seguridad Nacional y marcado por la influencia de los Estados Unidos sobre el Ejército y el Estado colombiano, se convirtió en la directriz política que orientó el comportamiento militar durante cuatro décadas. En este contexto, la seguridad nacional se entendió bajo una amenaza permanente por parte de fuerzas nacionales e internacionales ligadas al comunismo. La participación de militares norteamericanos en las operaciones contra los núcleos campesinos de orientación comunista, iba en la línea de la política exterior colombiana. Durante este periodo, Colombia se mantuvo férreamente alineada con los Estados Unidos, apoyando la expulsión de Cuba del Sistema Interamericano, en enero de 1962.

La situación cubana posibilitó la concreción de la vieja pretensión de varios países latinoamericanos de que Estados Unidos, promoviera un plan de financiamiento que estimulara el desarrollo, a través de una masiva transferencia de recursos norte sur. En este marco, el presidente John F. Kennedy, lanzo la Alianza para el Progreso.

La consolidación de la revolución cubana, hizo que Washington, considerara que el fomento de programas económicos, acompañados de reformas sociales, abonaría el camino para el surgimiento de gobiernos democráticos, dejando sin campo de maniobra los impulsos del comunismo en América Latina. En cierta forma, la Alianza para el Progreso marcó el punto de culminación de la contradicción de intereses presentes entre Latinoamérica, que propendía por dar prioridad a los temas económicos de la agenda, y los Estados Unidos, que ponían énfasis en los aspectos de seguridad y defensa. La amenaza que se desprendía desde Cuba, sobre Latinoamérica, precipi- tó la cooperación del hemisferio en contra del comunismo. Colombia fue uno de los grandes beneficiarios de la Alianza para el Progreso. La estabilidad institucional generada por el régimen bipartidista (Frente Nacional), instalado en 1958, presentaba un escenario favorable para la recepción e inversión de recursos, más, cuando el pacto político vigente, subordinaba los asuntos políticos a los económicos, además del irrestricto compromiso colombiano en la contención del castrismo. (Rojas, 2010)

Según Mitchell, citado por Cepeda \& Pardo (1989), entre 1962 y 1973, Colombia recibió ayudas financieras por parte de los Estados Unidos, por un valor de 1.203.3 millones de dólares. El tratamiento privilegiado para con Colombia, se evidencio con la visita del presidente Kennedy a Bogotá, en diciembre de 1961. En este contexto, Colombia comenzó a moverse hacia nuevos espacios internacionales, reanudando en 1968 sus vínculos diplomáticos con la Unión Soviética ${ }^{8}$, y según Cepeda \& Pardo (1989), mostró una nueva actitud frente a los acontecimientos mundiales, motivado en cierta forma, por los pobres resultados económicos de su leal amistad con los Estados Unidos. Durante el gobierno del presidente Carlos Lleras Restrepo (1966-1970), su canciller Alfonso López Michelsen, lideró un giro a las relaciones internacionales de Colombia, al buscar estrechar las relaciones con los Estados vecinos. De esta manera se produjo una primera ruptura en la relación de alineamiento político con los Estados Unidos, al sustituir al Réspice Polum por la "Réspice Similia", que significa "mirar a tus semejantes". De esta manera dio impulsó a un proceso de integración regional por medio del Pacto Andino, e inicio su participación, aunque limitada en la UNCTAD. (Conferencia de las Naciones Unidas sobre Comercio y Desarrollo)

\footnotetext{
${ }^{8}$ Vínculos que se rompieron tras el asesinato de Jorge Eliécer Gaitán en 1948.
} 


\section{Más comercio, pragmatismo y menos ideología}

Los hechos antes señalados marcaron el comienzo de una fase en las Relaciones Internacionales colombianas, en la que los intercambios comerciales se pusieron por sobre los gobiernos o el sistema político de los Estados. La administración del presidente Misael Pastrana (1970-1974), dio pasos en ese sentido. En 1973, el gobierno Pastrana, repudió el golpe de estado que instaló la dictadura de Augusto Pinochet en Chile, y defendió activamente el derecho de asilo, abriendo las puertas del país a todos aquellos chilenos que se sintieran perseguidos por la dictadura. De esta manera, la política exterior colombiana iniciaba un gradual proceso de desideologización y apertura de su diplomacia.

\section{Alfonso López Michelsen yel "Respicesimilia" (1974-1978)}

Durante la presidencia del liberal Alfonso López Michelsen, se presentó la que sería la primera ruptura frente a la política exterior de los Estados Unidos, siguiendo los lineamientos del "Réspice Similia", buscando relacionarse con Estados en niveles de desarrollo similares a Colombia. Según González (2004), se trataba de que en el ajedrez mundial, Colombia dejara de ser un peón de la guerra fría. En este sentido, López Michelsen, se propuso universalizar la política internacional colombiana, que en ese momento estaba marcada por los signos económicos, de ahí que la administración López, intentará rediseñar la política internacional, buscando la emancipación económica del Estado colombiano. Pruebas de esta nueva actitud en el escenario internacional, fueron la determinación de no aceptar exigencias en materias de políticas económicas por parte de organismos crediticios internacionales, el restablecimiento de relaciones diplomáticas con Cuba, el abierto apoyo a la suscripción del tratado Torrijos-Carter, que buscaba que Panamá asumiera el control del canal interoceánico administrado por los Estados Unidos.

En el informe que el presidente López, presentó al Congreso, el 20 de julio de 1978 al final de su periodo de gobierno manifestó que "había tomado ímpetu para la lucha para obtener en la mesa de negociación internacional lo que nuestras masas desposeídas reclamaban a nivel doméstico, es decir, igualdad de oportunidades y no caridad, y no filantropía. Justicia en las relaciones económicas y no ayudas". (López, 1978: 151)

\section{Julio Cesar Turbay Ayala (1978-1982). El retorno al anticomunismo}

La gestión del presidente Turbay, se inició, por una parte, en medio de un complejo panorama social y político marcado por la internacionalización del conflicto armado. Por otra, en el plano internacional, un escenario centroamericano en el cual Nicaragua, El Salvador y Granada, lideradas por Cuba, presentaban nuevos retos para los EEUU y sus aliados. De esta forma, se fue marcando un retorno al Réspice Polum en el manejo de la política exterior colombiana, aunque se mantuvieron ciertas líneas de apertura iniciadas durante la administración López, nuevamente el anticomunismo como ideología, se convertía en un rasgo central de la política internacional colombiana. El temor que despertaba en las elites políticas, económicas y militares de Bogotá y Washington, el avance del "comunismo soviético" en el Caribe, incidió directamente en el accionar internacional de ambos estados. El presidente Reagan, actúo con la decisión de alejar la amenaza cubano-soviética de la frontera sur estadounidense. Una de sus primeras 
acciones fue acusar abiertamente a $\mathrm{Ni}$ caragua de servir como base de acopio y distribución de armas soviéticas a los insurgentes salvadoreños y señalo a Cuba de proveer asesores y armas a las organizaciones guerrilleras Centroamericanas. Acto seguido, los Estados Unidos, financiaron, armaron y asesoraron las guerrillas antisandinistas (la contra), coordinó y dirigió maniobras militares desde y con Honduras, boicoteo la economía de Nicaragua, minando sus puertos e invadió la Isla de Granada en 1983. (Atkins, 1991)

Por su parte, Colombia sentía que podía ser blanco de la subversión que avanzaba en Centroamérica. Para Deas (1988), tanto la insurgencia como el Ejército colombiano, seguían con interés el desarrollo de los hechos en América Central, ya que un triunfo o derrota guerrillera en El Salvador, produciría indudablemente un "efecto dominó" en Colombia. En este sentido, dentro de las acciones desplegadas por la diplomacia colombiana, se destacan la oposición a la candidatura de Cuba al Consejo de Seguridad de la Organización de las Naciones Unidas (ONU) en 1979, la ruptura de relaciones diplomáticas con ese Estado en 1981, las conversaciones entre militares colombianos y estadounidenses en 1982, sobre la posibilidad de instalar una base especial en la Isla de San Andrés; ese mismo año Colombia participó en la "Comunidad Democrática Centroamericana”, cuyo objetivo era la defensa diplomática del gobierno salvadoreño y el ataque a la revolución sandinista (Tokatlian, 1999); Colombia también rechazó la declaración franco-mexicana, que recomendaba la negociación entre el gobierno y la guerrilla salvadoreña; apoyo a Inglaterra en la guerra de las Malvinas ${ }^{9}$, también envío tropas a la fuerza de pacificación del Sinaí.

\footnotetext{
${ }^{9}$ Varios países del continente repudiaron esta postura contra Argentina, llegando Colombia a ser tildada como el Caín latinoamericano.
}

\section{Belisario Betancur (1982-1986). De la independencia relativa a la dependencia involuntaria}

Durante los dos primeros años del gobierno de Belisario Betancur Cuartas, se da otro punto de quiebre respecto al alineamiento con Washington. En su momento, fueron muy comentadas las declaraciones que el presidente Betancur, entrego a la revista Newsweek (23 de agosto de 1982), citadas por González (2004), en las que afirmó que: "el gobierno de Washington trataba con desdén a Colombia, y añadió que nuestro país no deseaba ser satélite de ninguna superpotencia ni de Estados Unidos" (p. 269). De hecho, en diciembre de 1982 durante la visita realizada por el presidente de los Estados Unidos Ronald Reagan, a Bogotá, el presidente Betancur, hizo críticas al orden económico mundial y al papel desempeñado por los Estados Unidos en él. Propuso una nueva alianza social sin dependencias de ningún tipo. (Cepeda \& Pardo, 1989)

El gobierno colombiano desplegó una gestión internacional que buscaba complementar su labor interna en favor del diálogo y la salida política negociada al conflicto armado. La administración Betancur, sostenía que el vínculo entre el conflicto armado interno y la guerra en Centroamérica era directo, por lo cual le apostó a la búsqueda de la paz negociada en ambos frentes. De ahí, que se desprendiera de un elemento que había sido clave en la política exterior del gobierno anterior: el anticomunismo, sin esta decisión era imposible el inicio de diálogos que condujeran a escenarios de reconciliación con la insurgencia colombiana. En esta línea, Colombia asumió audaces posturas en el plano internacional al incorporarse de lleno en el grupo de los No Alineados, al buscar multilateralmente salida a problemas de la región como la deuda externa, la que entrelazó 
con la promoción del desarrollo económico sostenido y equilibrado que permitiera la superación de la pobreza y posibilitara la estabilidad político-institucional. También se involucró activamente en la búsqueda de la paz en Centroamérica, al ser miembro del "Grupo de Contadora", conformado por México, Panamá, Venezuela y Colombia, en enero de 1983.

El grupo propuso mediar en la negociación de la resolución pacífica del conflicto centroamericano. Redacto un listado de veintiún principios destinados a posibilitar los diálogos entre Nicaragua y los Estados Unidos y, entre la insurgencia y el gobierno salvadoreño. Dentro de los principios se destacan: Limitar el flujo de armamentos, retirar los asesores extranjeros (cubanos, estadounidenses y de Europa Oriental), prohibir el establecimiento de bases extranjeras y suspender el apoyo a los insurgentes. (Atkins, 1991)

Esta nueva diplomacia por la paz, como la denominó Tokatlian (1999), no se agotó en el contexto centroamericano, ya que en 1983 durante la invasión de los Estados Unidos a Granada, Colombia fue mediador en el proceso que permitió la salida del destacamento cubano desde la isla agredida. Gracias a esta acción, Fidel Castro remitió dos cartas a la guerrilla colombiana del ELN, para que liberara de manera unilateral e inmediata (como sucedió), al hermano del presidente Belisario Betancur, Jaime Betancur.

Para la segunda mitad de su mandato, las dificultades internas en torno a la concreción de la paz, el creciente poder político y económico del narcotráfico y la imposibilidad de evitar la influencia de los Estados Unidos en los asuntos de seguridad interna, fueron minando su autonomía en el plano internacional. Según Tokatlian (1999), cuando en 1984 el embajador de los Estados Unidos en Colombia,
Lewis Tambs acuñó el concepto "narcoguerrilla" para definir la naciente alianza entre guerrilla y narcotráfico, buscaba eliminar cualquier frontera que existiera entre la estrategia de paz (contrainsurgente) del gobierno colombiano y la percepción de amenaza que el narcotráfico representaba para la región.

El asesinato en 1984 del Ministro de Justicia, Rodrigo Lara Bonilla condujo a que Colombia cediera ante las presiones de los Estados Unidos y en 1985 implantara la extradición. La crisis de endeudamiento interno, terminó de acercar a la administración Betancur a los Estados Unidos, ya que la mediación de Washington ante el FMI era fundamental para la obtención de los recursos que necesitaba. Por último, la toma del Palacio de Justicia en noviembre de 1985 por parte del Movimiento Guerrillero 19 de Abril (M-19), condujo al gobierno colombiano a un cambio drástico en el manejo del orden público asumiendo una posición de mano dura contra la insurgencia. En este contexto, el presidente Betancur, definió al M-19 como "un movimiento portador de un proyecto terrorista”. (Tokatlian, 1999: 311-312)

\section{Virgilio Barco (1984-1988). El reencuentro con el Tercer Mundo \\ y la emergencia del narcotráfico}

El gobierno del liberal Virgilio Barco Vargas, inicio su actividad internacional realizando esfuerzos por diversificar las relaciones internacionales colombianas, tratando de evitar las consideraciones ideológicas e impulsando las relaciones comerciales y económicas con la mayoría de estados y bloques regionales posibles. Para este gobierno las relaciones internacionales debían ser conducidas por principios económicos y no políticos (González, 2004). La administración Barco, si bien 
no rompió totalmente con la tradición de alineamiento con los Estados Unidos, si generó un nuevo estilo en el manejo de la política internacional. En cierta forma, la estrecha coincidencia con Washington, en torno al tema de las drogas ilícitas le permitía al gobierno colombiano mantener diferencias en otros temas de la agenda binacional.

Al respecto Tokatlian (1994), sostiene que el disenso político no alteró el consenso económico con E.E.U.U. La actividad internacional del gobierno Barco, se construyó sobre tres ejes centrales: el primero consistió en descentroamericanizar la política exterior, buscando nuevos espacios en el Pacifico, el segundo se basó en tener un especial cuidado en la relación con los Estados Unidos y el tercero, buscaba propiciar por medio de un manejo conservador de la economía una mejoría sustancial en sus tratos con los organismos económicos multilaterales, aunque esto limitara sustancialmente su autonomía financiera. La administración Barco, consideraba que Colombia debía preparase para la nueva realidad internacional, por lo cual, el énfasis de su política exterior tenía que estar en la Cuenca del Pacifico. En paralelo, Colombia se mantuvo dentro de los No Alineados, tratando de mantener alguna autonomía frente a Washington. (Cardona, 1990)

Como prueba de su relativa autonomía internacional, Colombia mantuvo y amplío los contactos iniciados durante el gobierno anterior con Cuba, a pesar de que los contactos diplomáticos formales estaban suspendidos desde 1981, los puntos de encuentro entre ambos países se hicieron frecuentes. Fruto del mejoramiento en las relaciones binacionales, Colombia y Cuba, suscribieron el 12 de diciembre en la ciudad de Barranquilla, un Acuerdo de alcance parcial por medio del cual ambos países pretendían incrementar su inter- cambio comercial otorgando preferencias arancelarias reciprocas. Callejas \& González (1998), señalan que la relación con Cuba, no solo se dio en plano bilateral, también se proyectó en escenarios multilaterales, tal como sucedió en 1989 en el marco de la Comisión de Derechos Humanos de las Naciones Unidas, en la que Colombia trato por medio de abstenciones y votos en contra de los Estados Unidos, evitar una condena política contra el gobierno de la isla, por la situación interna de Derechos Humanos. Un año después ambos países coincidieron en el Consejo de Seguridad de las Naciones Unidas, y junto a Malasia y Yemen, conformaron el Grupo de los Cuatro, en el cual unificaron posiciones frente a la crisis del golfo pérsico, la independencia de Namibia, los territorios árabes ocupados por Israel y la invasión de los Estados Unidos a Panamá.

\section{Cesar Gaviria Trujillo (1990-1994). Un nuevo escenario internacional con los mismos problemas internos}

La administración del Presidente Gaviria, se inicia con el declive y terminación de la guerra fría como telón de fondo. Este nuevo escenario internacional abría posibilidades de acercamiento entre estados históricamente lejanos en sus relaciones diplomáticas. Dentro de los propósitos más relevantes en materia de política internacional de este gobierno, se encontraban: la ampliación de los intercambios comerciales con diversos estados, la internacionalización de la economía colombiana y la búsqueda del fortalecimiento de la capacidad negociadora de la región. Para Cardona \& Tokatlian (1991), el presidente Gaviria, dio continuidad a las políticas internacionales del gobierno anterior, al conservar a varios funcionarios de la antigua administración y mantener el énfasis en los temas económicos, buscando una mayor apertu- 
ra hacia el exterior, defendiendo siempre el derecho y las instituciones internacionales. En cuanto al tema del conflicto interno y la paz, el presidente Gaviria, reconocía la estrecha conexión existente entre la política internacional y la paz interna.

En este sentido, Colombia junto a España, México y Venezuela conformaron, con la anuencia del Secretario General de la ONU, y con el visto bueno de los Estados Unidos, el Grupo de Apoyo al proceso de Paz en El Salvador, que condujo al acuerdo definitivo de paz en 1992; y durante la Firma del Acuerdo Marco entre la Unidad Revolucionaria Nacional Guatemalteca (URNG) y el gobierno de Guatemala, las partes solicitaron que Colombia, España, Estados Unidos, México y Venezuela conformaran el grupo de países amigos del proceso de paz guatemalteco. Además, Colombia buscó en la Organización de los Estados Americanos (OEA) la restitución en el poder del Presidente electo de Haití, Jean Bertrand Aristide, quien había sido derrocado por un golpe de estado en 1991 (Tokatlian, 1999). En cuanto a los vínculos con Cuba, afianzados durante la administración anterior, finalmente en octubre de 1991 se reactivaron las relaciones diplomáticas formales entre Colombia y la isla. Este hecho, impacto directamente en la situación de orden público y los derechos humanos en Colombia.

En el plano multilateral, Colombia se vinculó a la Asociación de Estados del Caribe (AEC), en julio de 1994 y la obtuvo la presidencia del grupo de los países No Alineados. Al final de su mandato, el narcotráfico y los derechos humanos pasaron al centro de la agenda internacional, lo que limitó sus márgenes externos de maniobra, ya que necesitaba de los Estados Unidos, para la contención y resolución del complejo problema de las drogas, que para ese momento amenazaba seriamente la institucionalidad del Estado colombiano.
Según Garay, Angulo \& Cadena (1994), en el plano económico, Washington jugó un papel determinante en la consecución del crédito Hércules por un valor de US\$ 1.775 millones de dólares para el periodo 1991-1994.

\section{Ernesto Samper (1994-1998).}

\section{La narcotización de las relaciones exteriores}

La administración del Presidente liberal Ernesto Samper Pizano (1994-1998), se inició con serios tropiezos en sus relaciones con los Estados Unidos, en gran medida por el escándalo que produjo la confirmación del ingreso de dineros del narcotráfico (Cartel de Cali) a su campaña presidencial. En el plano interno, la agravación sostenida de las violaciones a los derechos humanos, hizo que la comunidad internacional percibiera esta situación como un signo de debilidad estatal. En 1995, Samper asumió la presidencia del movimiento de los países No Alineados. Lo que se presentaba como una oportunidad de adquirir reconocimiento y dinamismo en su actividad internacional, se subvaloró y dilapidó entre otras cosas por la falta de análisis y planeación sobre los verdaderos alcances, costos y beneficios de esta responsabilidad internacional, la ausencia de un consenso político interno y por el estigma que siguió al movimiento desde el momento de su fundación. Según Socorro Ramírez, citada por González (2004):

el país se vio embarcado en la mayor responsabilidad de su historia sin que la decisión hubiera sido previamente debatida por nadie y sin que ningún sector de la sociedad, ni el mismo gobierno, supiera con claridad las consecuencias, sus costos o beneficios, desde luego, sin plan ni estrategia premeditada para rentabilizar a favor de los intereses nacionales (p. 278). 
Según Rojas (2006), la crisis del gobierno Samper, evidenció hasta qué punto el narcotráfico se había instalado en la sociedad colombiana lo que encendió las alarmas en los Estados Unidos sobre la real posibilidad de estar en presencia de un Estado controlado por el crimen organizado. La creciente importancia de Colombia en la economía global de la cocaína y el aumento sostenido de los vínculos entre organizaciones armadas con la economía de la droga, fueron escalando el conflicto armado, lo que condujo a que los Estados Unidos aplicase a fondo la estrategia antinarcóticos afectando a diversas poblaciones por las fumigaciones aéreas (Vargas, 1999). Durante la administración Samper, los Estados Unidos buscaron evitar que Colombia se convirtiera en una narco democracia, llegando por primera vez en más de treinta años de conflicto armado a deslegitimar públicamente a un gobierno colombiano. El hecho de cancelarle la visa estadounidense al presidente Samper, la descertificación en los años 1996 y 1997.

Para el final de la administración Samper, el Departamento de Defensa norteamericano, afirmaba que las organizaciones armadas debido a su relación con el narcotráfico habían alcanzado altos niveles de sofisticación y representaban la mayor amenaza a los militares colombianos. Desde ese momento la agenda bilateral si bien mantenía el problema de las drogas como eje central, empezaba a ser atravesada con mayor frecuencia por el conflicto armado. (Rojas, 2006)

\section{Andrés Pastrana (1994-1998).}

\section{De la ayuda antinarcóticos a la intervención} internacional en el conflicto armado colombiano

La administración del presidente Andrés Pastrana Arango (1994-1998), se inició con un proceso de negociación política con la guerrilla de las FARC-EP. La estrategia de paz de este gobierno buscaba la máxima participación de la comunidad internacional en el proceso de negociación, poniendo su énfasis en los Estados Unidos. Esta situación se evidenció durante la visita que efectúo el presidente Pastrana a Washington en octubre de 1998, en esta reunión ambos mandatarios acordaron la "Alianza contra las drogas ilícitas", esta sería la base de las relaciones binacionales durante este cuatrienio. Hasta ese momento, el Departamento de Estado afirmaba que era posible replicar en Colombia el marco estratégico utilizado en El Salvador a fines de los ochenta. Desde esta perspectiva se evita la participación directa de tropas y se promueve la asistencia técnica sostenida por medio de entrenamiento, tecnología de inteligencia y equipos especializados, con el objetivo de debilitar a la insurgencia y forzar su decisión de negociar.

El apoyo de los Estados Unidos, a la negociación de paz, se afectó como consecuencia del asesinato de tres indigenistas norteamericanos a manos de las FARCEP en marzo de 1999. La postura de Washington, con respecto a las FARC-EP se hizo manifiesta por medio de la visita en agosto de 1999 de Thomas Pickerin quien manifestó la preocupación de su gobierno con respecto a la forma en que se estaba conduciendo la "Zona de Despeje" y los abusos que ahí se estaban presentando. Desde ese momento, los Estados Unidos condicionaron la continuación de su apoyo a Colombia ${ }^{10}$, al diseño de un plan coherente para la lucha contra el narcotráfico, a lo que el gobierno colombiano accedió sin condiciones. (González, 2004)

\footnotetext{
${ }^{10}$ En 1999, Colombia era el tercer país del mundo en recibir más ayuda militar de los Estados Unidos.
} 
De esta manera, el Plan Colombia que según Rojas (2006), en 1998 había sido presentado por el mandatario colombiano, como una especie de Plan Marshall para el desarrollo económico y social pasó rápidamente a ser la base de la estrategia antidrogas de los Estados Unidos, para toda la región andina. El Plan Colombia combinó medidas antidrogas como el control de precursores químicos, la fumigación de cultivos ilícitos, destrucción de laboratorios, decomiso de cargamentos e interdicción aérea; y estrategias de recuperación del control estatal de las zonas productoras de droga. De ahí, que en su primera fase, el Plan se dirigiera hacia los departamentos de Putumayo y Caquetá, principales productores de cocaína y militarmente controlados por el Bloque Sur de las FARC-EP. En el despliegue territorial del Plan, jugaban un papel fundamental los batallones militares antinarcóticos, entrenados y equipados por los Estados Unidos. Estos, pasaron a ser el componente militar de la política antidroga. En la práctica, el Plan Colombia se convirtió en el primer paso del involucramiento directo de los Estados Unidos, en el conflicto armado colombiano, ya que a pesar de manejar con relativa cautela la autonomía de Colombia respecto al manejo de su conflicto interno, hay temas de su agenda propia en Colombia, que fueron afectados por el conflicto armado. Temas como el narcotráfico, la democracia, los derechos humanos, y la falta de garantías de seguridad para sus inversiones, se vieron cada vez más impactados por el escalamiento y degradación de la guerra en Colombia (Rangel, 2001). No fueron pocas las críticas que despertó el Plan Colombia y su aplicación, por su carácter militarista e intervencionista, por ejemplo el Parlamento Europeo en su declaración de febrero de 2001, citada por González (2004), expresó su total respaldo al proceso de paz y a la solución concertada del conflicto, reconociendo las dimensiones políticas y sociales del mismo.

En la misma línea, Pinto (2001), se refería a la aplicación del Plan Colombia, desde el punto de vista de algunos voceros del gobierno venezolano, los que en diversas oportunidades habían hecho referencia a que el Plan Colombia podía provocar un desequilibrio militar en la región andina, particularmente entre Colombia y Venezuela. La cooperación militar con los Estados Unidos, durante el gobierno Pastrana, en palabras del propio ex presidente "se tradujo en la actualización de la aviación existente, se mejoraron los sistemas de aviónica de los helicópteros de la armada y se doto un avión de reconocimiento". (Vargas, 2010:137)

\section{Álvaro Uribe Vélez (2002-2010). La securitización de la política exterior colombiana}

El primer triunfo presidencial de Álvaro Uribe Vélez (2002-2006), estuvo marcado por las siguientes variables socio-políticas, variables que contextualizaron el inicio de su administración:

Terminación por parte de la administración Pastrana de las conversaciones de paz; Un escenario internacional globalizado, con los Estados Unidos, como hegemón político y militar dominante, instalando una nueva agenda internacional, centrada en la lucha global contra el terrorismo. Y una Región Andina, inestable institucionalmente y cambiante en lo político.

\section{La política de Defensa y Seguridad Democrática}

La entonces Ministra de Defensa Martha Lucia Ramírez, citada en el informe del Programa de las Naciones Unidas para el Desarrollo - PNUD (2003), definía la Política de Defensa y Seguridad Democrática 
(PDSD), como una política estatal de largo plazo para proteger a la población. La primera estrategia seguida por el Estado, fue la de contener y proteger; Teniendo como punto de partida la protección del libre tránsito vehicular por las principales vías del país, brindando seguridad a los viajeros a través de caravanas militares de escolta, llamadas "Vive Colombia, viaja por ella", y la puesta en marcha del Plan Meteoro. Al mismo tiempo, se inició la recuperación del control territorial. Según Pizarro (2004), al inicio de la administración Uribe, la Policía Nacional se encontraba ausente de 160 municipios, aproximadamente el $15 \%$ de todos los municipios colombianos. En el segundo semestre de 2003 sólo quedaba un 5\% de municipios sin presencia estatal, y en el año 2004, había presencia permanente de la Policía Nacional en todas las cabeceras municipales de Colombia. (Vargas, 2010)

En el plano internacional la primera medida de la administración Uribe, fue ubicar el conflicto armado colombiano dentro de la "cruzada mundial contra el terrorismo" liderada por los Estados Unidos, después de los atentados del 11 de septiembre. En un contexto internacional marcado por el posicionamiento del terrorismo como la principal amenaza de la agenda de seguridad mundial, el presidente Uribe, logró presentar el conflicto armado colombiano como una amenaza no solo para la región, ya que al ser las FARC-EP, el ELN y las Autodefensas Unidas de Colombia (AUC), consideradas organizaciones terroristas por el Departamento de Estado norteamericano, y además al estar involucradas con el tráfico de narcóticos, el alcance de sus acciones armadas adquiría dimensiones globales. En este caso, puede apreciarse con claridad la relación presente entre la política interna y la política externa del presidente Uribe. Relación, que por la longevidad y degradación del conflicto armado colombiano, adquiere relevancia.
En este sentido, según Tokatlian (1999), Todo estado, en cuyo territorio se desarrolla una guerra, dirige parte de su diplomacia a la búsqueda de apoyo externo para la resolución de su guerra interna. En el caso colombiano, la diplomacia de la administración Uribe, buscó legitimar internacionalmente la utilización por parte del Estado colombiano, de un mayor uso de la fuerza, tendiente a la derrota política y militar de la insurgencia y el narcotráfico. De esta manera, el tema de la seguridad, se convirtió en el eje sobre el cual gravitó la política exterior del presidente Uribe. Desde un enfoque teórico, la diplomacia del gobierno Uribe, se encuadró en la escuela Neorrealista de las relaciones internacionales, ya que la seguridad se convirtió en el tema central de su accionar político. En este marco, la seguridad, además de ser la base sobre la cual se edificó su gobierno, pasó a ser la piedra angular del proceso de la toma de decisiones de su política exterior, es decir, se securitizó la misma. (Jiménez, 2004)

De tal manera que la adscripción del conflicto armado colombiano en la lucha mundial contra el terrorismo, y su alianza incondicional con los Estados Unidos, resultó exitosa, ya que Colombia, mantuvo y consolidó la ayuda militar de los Estados Unidos, a través del Plan Colombia, asegurando de esta manera la sostenibilidad del componente militar de la Seguridad Democrática. Fue así, como el Plan Colombia, pasó de ser un plan antinarcóticos en su planeación, a un plan antiterrorista, en su denominación y a un plan contrainsurgente, en su ejecución, es decir, el esfuerzo de la administración Uribe, se dirigió a incrementar la ayuda económica de los Estados Unidos, para mejorar la capacidad disuasiva de las Fuerzas Armadas y ponerlas en actitud ofensiva frente a la insurgencia. 


\section{RESULTADOS}

La política exterior de Colombia, ha sido marcada profundamente por el intervencionismo de los Estados Unidos desde el pronunciamiento de Marco Fidel Suarez a partir de los inicios de la década del 20 del siglo pasado, mediante la expresión del Réspice Polum.

En tal sentido, el país Suramericano fue el principal beneficiario de la Alianza para el Progreso y paralelamente la cúpula militar colombiana se formó bajo las directrices de los norteamericanos en la Escuela de las Américas lo que sin duda, fue determinante en el ejercicio de interacción con el resto del sistema en el escenario bipolar.

Con el derrumbe de la guerra fría, Colombia, a diferencia de la mayor parte de la región, continuó por la senda de la "estrella del norte", invitándolo a participar como actor relevante del conflicto, mediante la implementación del Plan Colombia, con lo cual, se escaló hacia una securitización en la relación con los otros actores de la región, lo que condujo a una creciente tensión.

En este orden de ideas, el periodo de mayor securitización, repercutió negativamente en la relación económica y comercial, denotando un ostensible descenso en las exportaciones hacia tradicionales socios estratégicos como Ecuador y Venezuela. Se demuestra a lo largo del análisis que la necesidad del mercado norteamericano y los empréstitos de la banca, sumado a los problemas de orden interno como narcotráfico, subversión y terrorismo han tejido una dependencia casi estructural con Norteamérica lo cual contrasta con el resto de los países de la región.

\section{CONCLUSIONES}

Del anterior análisis puede afirmarse que si bien la política exterior colombiana ha buscado la universalización de su diplomacia, la relación con los Estados Unidos ha sido el eje sobre el cual ha gravitado su actividad internacional, limitándola o condicionándola, en lo económico, lo político y en temas de seguridad interna. Si bien esta dependencia ha sido continua, ha habido intentos de generar rupturas, pero distintos factores han mantenido en el tiempo dicha continuidad, teniendo como referente el Respice Polum. En este proceso de dependencia, se pueden encontrar cuatro momentos diferentes con un eje específico.

Un primer momento fundado en el anticomunismo (1948 - 1970), un segundo momento marcado por la necesidad de mercados y empréstitos (1974 - 1990), un tercer momento centrado en el combate al narcotráfico (1990 - 2002) y un cuarto momento enfocado en la lucha global contra el terrorismo (2002 - 2010). Las consecuencias más directas de esta situación fueron:

a. El marcado distanciamiento de la región y la desconfianza de sus vecinos, impactando directamente las condiciones migratorias de sus nacionales y a un sector importante de sus exportaciones;

b. La violación del Derecho Inter-nacional, como sucedió en el ataque al campamento del comandante guerrillero Raúl Reyes, en la selva ecuatoriana;

c. El descuido y subvaloración de importantes espacios políticos y comerciales, como Europa y América Latina y el Caribe, precisamente los espacios, en los que la actividad internacional de las FARCEP tuvo sus mayores desarrollos. 
Es decir, la diplomacia de las FARC-EP no tuvo contrapeso estatal. Tal y como lo manifestó el ex presidente Álvaro Uribe Vélez, en abril de 2009: "Tuvieron una gran diplomacia internacional que superó a la cancillería colombiana porque no se les enfrentó debidamente".

Esta situación se ha visto agravada entre otros factores por el marcado personalismo con que los presidentes colombianos han encarado la política exterior del país, lo que evidencia la ausencia de una política de estado que persiga objetivos internacionales de largo plazo. Hemos observado como a lo largo de la guerra fría nuestro país ha estado orientado a asumir como suyas las políticas de los Estados Unidos, motivo por el cual la política exterior ha girado en función de manejar la agenda política y económica en función de los intereses estratégicos de la Casa Blanca. Por tanto hemos sido un socio estratégico y a la vez un aliado incondicional del mundo capitalista, incluso después de la finalización del conflicto Este- Oeste.

\section{REFERENCIAS}

Atkins, G. (1991). América Latina en el sistema politico internacional. Buenos Aires: GEL.

Callejas \& González, R. (1998). Colombia y Cuba. Del distanciamiento a la cooperación. Barranquilla: Ediciones Uninorte.

Cardona, D. (1990). La administración Barco y los No Alineados, Revista Colombia Internacional, 9, 3-9.

Cardona, D. \& Tokatlian, J. (1991). Los desafíos de la política internacional Colombiana en los noventas, Revista Colombia Internacional, 14

Cepeda, F. \& Pardo, R. (1989).La política exterior colombiana (1974-1986). Bogotá: Editorial Planeta.
Deas, M. (1988). El proceso de paz colombiano, 1982-1985 y sus implicaciones para Centroamérica, Documentos Ocasionales, 5 .

Garay, L., Angulo, A. \& Cadena, C. (1994) Cultura de negociación: la experiencia de la deuda externa. Bogotá: CEREC-FESCOL.

González, R. (2004). "La política exterior de Colombia a finales del siglo XX. Primera aproximación", Investigación y Desarrollo, 12(2), 358-385.

Jiménez, N. (2004). "Implicaciones de la securitización en América desde la Particularidad colombiana”. Revista Investigación y Desarrollo, 12(2), 244-257.

López, A. (1978). Testimonio Final. Bogotá: Imprenta Nacional.

Meléndez, J. (2014). Colombia y su participación en la guerra de Corea. Una reflexión tras 64 años de iniciado el conflicto, Revista Historia y memoria, 10(1), 199-239.

Pardo, R. (1987). La política exterior de la administración Barco. Análisis Politico, 2, 3-9.

Pinto, M. (2001). "El Plan Colombia y los Procesos de Integración Subregional Andina. Visión desde Venezuela", Universidad Central de Venezuela, Ponencia preparada para ser presentada en el XXIII Congreso Latín American Studies Association. Washington, D.C.

Pizarro, E. (2004). Una democracia asediada. Balance y perspectivas del conflicto armado en Colombia. Bogotá: Grupo Editorial Norma.

Rangel, A. (2001). Guerra contrainsurgente, conflictos armados en Malasia, Perú, Filipinas, El Salvador y Colombia. Bogotá: Intermedio Editores.

Rojas, D. (2006). Los Estados Unidos y la guerra en Colombia. Bogotá: Grupo Editorial Norma. 
Rojas, D. (2010). La Alianza para el Progreso en Colombia. Recuperado de: http://www.ceecolombia.org/ ckfinder/userfiles/files/Ponencia\%20 APP\%20 Congreso\%20RRII\%20 \%28Diana\%20Rojas\%29.pdf

Tokatlian, J. (1994). "La política exterior del gobierno del presidente Virgilio Barco: En busca de la autonomía perdida" en: El gobierno de Barco. Política, economía y desarrollo social en Colombia, 1986-1990. Bogotá: Fedesarrollo - Fondo Cultural Cafetero.
Tokatlian, J. (1999). "Las diplomacias por la paz" Armar la paz es desarmar la guerra. Herramientas para lograr la paz. Bogotá: FESCOL, IEPRI, CEREC.

Vargas, A. (2010). "Seguridad Democrática, conflicto interno armado y su regionalización" Seguridad en Democracia; Un reto a la violencia en América Latina. Buenos Aires: CLACSO, Colección Grupos de Trabajo.

Vargas, R. (1999). Drogas, máscaras y juegos. Narcotráfico y conflicto armado. Bogotá: Tercer Mundo Editores. 


\title{
UNITED STATES IN COLOMBIAN FOREIGN POLICY: ARE THEY A STAUNCH ALLY? ${ }^{1}$
}

\author{
Roberto González Arana² - Héctor Galeano David² - Luis Fernando Trejos Rosero ${ }^{4}$
}

Universidad del Norte, Colombia - Article Type 1.

Science and Technology Research - Received: September 2nd 2013 - Accepted: April 27th 2015

doi: http://dx.doi.org/10.17981/econcuc.36.1.2015.23

\begin{abstract}
This article analyzes the foreign policy of Colombia from 1950 to 2010 and US influence in its design and implementation. It shows how Colombia strengthened its status as a strategic partner framing its foreign policy in the doctrine called Réspice Polum ${ }^{5}$, being almost completely far from other options in economic and political matters, despite having made the Réspice Similia ${ }^{6}$. This study is qualitative, bibliographical and descriptive. It was demonstrated with this, the market need and loans during the early twentieth century and the inability of Colombia to control internal security problems. It was concluded that this has made an almost structural dependency in the relationship with the US, and that has been the axis around which Colombia's international activities in the economic, political and particularly on issues of internal security have turned.
\end{abstract}

\section{Keywords:}

Respice Polum, Respice Similia, Foreign Policy, Securitization.

JEL: F35, F50, F52

\section{If you need to reference this article.}

Gonzáles, R., Galeano, H., \& Trejos, L. (2015). United States in colombian foreign policy: Are they a staunch ally? Económicas CUC, 36(1), 79-106. doi: http://dx.doi.org/10.17981/econcuc.36.1.2015.23

\footnotetext{
${ }^{1}$ This article is a reflection from research "Colombian foreign policy during the twentieth century, objectives, means and strategies", which was developed under the CLACSO Working Group" Social movements and revolutionary movements in Central America and the Caribbean ".

${ }^{2} \mathrm{PhD}$ in History of the Russian Academy of Sciences. Professor in the Department of History and Social Sciences and Director of the Institute of Latin American and Caribbean Studies Universidad Del Norte. CLACSO working group coordinator, member of the research group Memories of the Caribbean, Universidad Del Norte. rogonzal@uninorte.edu.co .

${ }^{3}$ Universidad de la Costa. Professor and researcher in the area of international relations. hgaleano1@cuc.edu.co Masters in Conflict Resolution and Mediation, $\mathrm{PhD}$ (c) Social Sciences of the Universidad del Norte.

${ }^{4}$ American Studies doctor, major in International Studies (IDEA/USACH). Professor and researcher at the Department of Political Science and International Relations at Universidad del Norte. Research associate at the Institute of Caribbean and Latin American Studies, Universidad del Norte. Member of the Research Group "International Agenda" Universidad Del Norte. trejosl@uninorte.edu.co

${ }^{5}$ The motto Respice Polum (look to the North Star), became the axis of Colombian foreign policy. This policy sought to establish a deeper bilateral relation on trade issues; it was the context in which it has been developed a historic alignment of Colombia to the United States.

${ }^{6}$ Seeking relations with states of similar development levels to Colombia.
} 


\section{INTRODUCTION}

With the end of World War II, the global order came in transition towards a new world order that governed the rules of international politics until 1989. The bipolarity, as this period became known, urged states to align with one of the two "players", regarded as superpowers, i.e. the United States and the Union of Soviet Republics (USSR). Colombia was no exception to this process of rethinking of the global "arena". From this historic juncture, the country strengthens its unrestricted alliance with the United States, framing its foreign policy in the Respice Polum (look north) proposed by Marco Fidel Suarez in the 20s.

With the gradual construction of the new international system, local or regional conflicts emerged fueled by East-West tension. With the gradual construction of the new international system, local or regional conflicts, fueled by East-West tension they were developed. Not surprisingly, the United States enacted aimed at containing $^{7}$ the expansionist Soviet communism doctrines, which at the time had Eastern Europe as a priority. In this scenario, the first war of the Second World War breaks out; the Korean War. The war in the Far Asia, showed the position in foreign policy of Colombia from mid-century, set in total adherence to the United States. By decision of President Laureano Gomez, the Colombian military is the only one involved in the conflict in the Korean peninsula. According to Meléndez (2014), Colombian active participation began with decree 3230 of 1950 which was available shipping frigate "Almirante $\mathrm{Pa}$ -

\footnotetext{
${ }^{7}$ The containtment of the growing socialism in Europe becomes the main goal of US foreign policy. Doctrines such as Truman, Eisenhower and Reagan later focus their priorities on international security, curb the spread of socialism.
}

dilla" and decree 3927 of December 1950 the Infantry Battalion No. 1 Colombia was created, which would be trained in Bogotá and the United States and then join one of the US companies in the combat zone.

\section{DEVELOPMENT}

\section{The National Front (1958-1974)}

With the presidency of the Liberal Alberto Lleras Camargo (1958), the National Front (political and electoral coalition between the traditional, liberal and conservative parties) began. At that time, the doors of democracy were closed to all those groups, political parties or movements that were not liberal or conservative. Since then, the siege and the exclusion of third political forces would be a constant in the history of Colombia. For the government, the only way to establish the rule of peace on the international scene was to support unrestrainedly the actions of the United States.

According to Pardo (1987), in this way, Colombia, expressed its willingness to declare "international communism" as an enemy of the hemisphere, thus assuming, American interests as their own. Domestically, it is noteworthy regard to autonomous management (outside the civil control) that security and public order, did the Colombian Armed Forces. What President Lleras wanted, it was to keep the neutral military against the traditional parties and prevent the repetition of a coup. Thus, from 1958-1990 Lleras Doctrine was the only global policy guidance on military matters.

In the absence of a clear political strategy, which led the military effort of the state, anti-communism contained in the Doctrine of National Security and 
marked by the influence of the United States on the Army and the Colombian State, became the guideline which oriented military policy behavior for four decades. In this context, national security is under a permanent threat understood by national and international forces linked to communism. US military involvement in operations against communist peasant cores guidance was in line with Colombia's foreign policy. During this period, Colombia remained tightly aligned with the United States, supporting the expulsion of Cuba from the inter-American system, in January 1962.

The Cuban situation made possible the realization of the old claim of several Latin American countries that the United States, promoted a financing plan that would stimulate development through a massive transfer of resources north south. In this context, President John F. Kennedy launched the Alliance for Progress (Alianza para el Progreso). The consolidation of the Cuban Revolution, made Washington, consider that the promotion of economic programs, accompanied by social reforms, would pay the way for the emergence of democratic governments, leaving no room for maneuver impulses of communism in Latin America. In a way, the Alliance for Progress marked the completion point of the present conflict of interests between Latin America, which tended to give priority to economic issues on the agenda, and the United States, that emphasized safety aspects and defense. The threat was clear from Cuba on Latin America, precipitated hemispheric cooperation against communism. Colombia was one of the main beneficiaries of the Alliance for Progress. Institutional stability generated by the (National Front) two-party system, installed in 1958, had a favorable scenario for the receipt and investment of resources, plus, when the current political agreement, political affairs subordinated to economic, besides the Colombian unrestricted commitment in the containment of Castroism (Rojas, 2010).

According to Mitchell, quoted by Cepeda \& Pardo (1989), from 1962 to 1973, Colombia received financial aid from the United States, amounting to 1.203 .3 billion. The privileged treatment with Colombia was evidenced by the visit of President Kennedy to Bogota in December 1961. In this context, Colombia began to move into new international spaces, resuming in 1968 its diplomatic ties with the Soviet Union ${ }^{8}$, according Cepeda \& Pardo (1989), Colombia showed a new attitude to world events, somehow motivated by the poor economic performance of their loyal friendship with the United States. During the administration of President Carlos Lleras Restrepo (1966-1970), Foreign Minister Alfonso Lopez Michelsen, he led a turn for the international relations of Colombia, to seek closer relations with neighboring states. Thus it came the first break in the relationship of political alignment with the United States, replacing Réspice Polum by "Réspice Similia", which means "to look at your fellow man". Thus this gave impetus to a process of regional integration through the Andean Pact, , And initiated its participation, although limited in UNCTAD. (United Nations Conference on Trade and Development)

\section{More trade, pragmatism and less ideology}

The aforementioned facts marked the beginning of a phase in Colombia's International Relations, in which trade was considered to be more important than the government or the political system of states. The administration of President Misael Pastrana (1970-1974), took steps

\footnotetext{
${ }^{8}$ Ties that were broken after the assassination of Jorge Eliecer Gaitan in 1948.
} 
in that direction. In 1973, Pastrana's government condemned the coup that installed the dictatorship of Augusto Pinochet in Chile, and actively defended the right of asylum, opening the country's doors to all those Chileans who were feeling persecuted by the dictatorship. Thus, the Colombian foreign policy began a gradual process of de-ideologization and opening of its diplomacy.

\section{Alfonso López Michelsen \\ and the "Respice Similia" (1974-1978)}

During the presidency of the liberal Alfonso Lopez Michelsen, it was presented what would be the first break against the foreign policy of the United States, along the lines of "Similia Respice" seeking relations with States of similar levels of development to Colombia. According to Gonzalez (2004), it meant that in the chess world, Colombia stopped being a pawn in the Cold War.

In this sense, Lopez Michelsen, proposed to make Colombian foreign policy universal, which at that time was marked by economic signs, hence the Lopez administration, try to redesign the international politics, seeking the economic emancipation of the Colombian state. Evidence of this new attitude in the international arena, were the determinations of not accepting demands in matters of economic policies by international lending agencies, the restoration of diplomatic relations with Cuba, the open support to the signing of the Torrijos-Carter treaty which sought that Panama took over the canal administered by the United States.

The report that President Lopez, presented to Congress on July 20, 1978 at the end of his term in office said that "he had taken impetus to the fight for the international negotiating table what our dispossessed masses demanded at level domestic, that is, equal opportunities, not charity, not philanthropy. Justice in economic relations, not aid "(Lopez, 1978: 151).

\section{Julio Cesar Turbay Ayala (1978-1982).}

\section{The return to anticomunism}

The administration of President Turbay, was launched, firstly, in the midst of a complex social and political landscape marked by the internationalization of the armed conflict. Furthermore, at the international level, a Central American scenario in which Nicaragua, El Salvador and Granada, led by Cuba, presented new challenges for the US and its allies. Thus, it was marking a return to Respice Polum in the management of Colombian foreign policy, although certain opening lines initiated during the López administration remained again anti-communism as an ideology, became a central feature of international politics Colombian. The fear aroused in the political, economic and military elites of Bogota and Washington, the advance of "Soviet communism" in the Caribbean, had a direct impact in the international actions of both states. President Reagan, acted with the decision of removing the Cuban-Soviet threat in the southern US border. One of his first actions was accusing Nicaragua openly to serve as a basis for collection and distribution of Soviet arms to the Salvadoran insurgents and pointed to Cuba to provide advisers and weapons to Central American guerrilla organizations. Then, the United States, financed, armed and advised the anti- Sandinista guerrillas (the counter), coordinated and directed military exercises from and Honduras, boycotted the economy of Nicaragua, mining its harbors and invaded the island of Granada in 1983 (Atkins, 1991). 
On the other hand, Colombia felt it could be a target of the ongoing subversion in Central America. To Deas (1988), both the insurgency and the Colombian army, they followed with interest the development of events in Central America, since a guerrilla victory or defeat in $\mathrm{El}$ Salvador, in Colombia would undoubtedly produce a "domino effect.". "In this regard, within the actions undertaken by the Colombian diplomacy, the opposition to Cuba's candidacy to the Security Council of the United Nations (UN) in 1979 , the severance of diplomatic relations with that state in 1981, talks between military Colombian and US in 1982 on the possibility of installing a special base in San Andres Island are highlighted, Colombia participated in the "Central American Democratic Community", which aimed at diplomatic defense of the Salvadoran government and attacking the Sandinista revolution (Tokatlian, 1999); Colombia also rejected the Franco-Mexican declaration, that recommended the negotiation between the government and the Salvadoran guerrillas; support England in the Falklands War ${ }^{9}$, also sending troops to the peacekeeping force in Sinai.

\section{Belisario Betancur (1982-1986). \\ From relative independence to involuntary dependence}

During the first two years of Belisario Betancur Cuartas' government another break point is given regarding the alignment with Washington. At the time, the statements that President Betancur, gave to Newsweek (August 23, 1982) were very commented, quoted by González (2004), in which he stated that

9 Several contries of the continent repudiated this position against Argentina, calling Colombia Cain. "the US government was contemptuously to Colombia, adding that our country did not wish to be satellite of any superpower or the United States "(p. 269). In fact, in December 1982 during the visit by the President of the United States Ronald Reagan, to Bogota, President Betancur, was critical to world economic order and the role played by the United States in it. He proposed a new social alliance without any dependencies (Cepeda \& Pardo, 1989).

The Colombian government deployed an international management seeking to complement its domestic work for dialogue and a negotiated political solution to the armed conflict. Betancur's administration, argued that the link between armed conflict and war in Central America was direct, so he decided to search for a negotiated peace on both fronts. Hence, it is apparent from an element that was a key to the foreign policy of the previous government: anticommunism, without this decision was impossible to start dialogues that would lead scenarios to reconciliation with the Colombian insurgency. This way, Colombia took bold stances in the international arena to be fully incorporated into the group of the Non-Aligned, to multilaterally seek out problems in the region such as external debt, which intertwined with the promotion of sustainable and balanced economic development that would allow the elimination of poverty and enable the political and institutional stability. It was also actively involved in the search for peace in Central America, as a member of the "Contadora Group", made up of Mexico, Panama, Venezuela and Colombia, in January 1983. The group proposed to mediate in the negotiation of a peaceful resolution of the Central American conflict. They drafted a list of twenty principles to facilitate the dialogue between Nicaragua and the Unit- 
ed States and between the insurgency and the Salvadoran government. Among those principles were: Limiting the flow of arms, withdrawing foreign advisers (Cubans, Americans and Eastern Europe), prohibiting the establishment of foreign bases and stopping supporting insurgents (Atkins, 1991).

This new diplomacy for peace, as Tokatlian called it (1999), was not exhausted in the Central American context, as in 1983 during the invasion of the United States to Granada, Colombia was a mediator in the process that led to the departure of Cuban detachment from the island attacked. Thanks to this action, Fidel Castro sent two letters to the Colombian guerrillas of the ELN, to release unilaterally and immediately (as it happened), President Belisario Betancur's brother, Jaime Betancur.

For the second half of his term, internal difficulties surrounding the realization of peace, the growing political and economic power of drug trafficking and the inability to avoid the influence of the United States in matters of internal security, were undermining his autonomy at the international level. According Tokatlian (1999), when in 1984 the US ambassador in Colombia, Lewis Tambs coined the term "narco-guerrillas" to define the emerging alliance between guerrillas and drug trafficking, he sought to eliminate any border that existed between peace strategy (counterinsurgency) the Colombian government and the perceived threat that drug trafficking posed to the region.

The murder of Justice Minister in 1984 led to Colombia yielded to pressure from the United States and in 1985 implanted extradition. The internal debt crisis, made Betancur's administration closer to the United States, as Washington's mediation before the IMF was essential to obtain the resources it needed. Finally, the takeover of the Palace of Justice in November 1985 by the guerrilla April 19 Movement (M-19), led the Colombian government to a drastic change in the management of public order assuming a tough position against the insurgency. In this context, President Betancur, defined the M-19 as "a movement carrier of a terrorist project" (Tokatlian, 1999: 311-312).

\section{Virgilio Barco (1984-1988).}

The encounter with the Third World and the emergence of drug trafficking

Virgilio Barco Vargas' liberal government began its international activity making efforts to diversify the Colombian international relations, trying to avoid ideological considerations and boosting trade and economic relations with most states and potential regional trade blocs. According to this government, international relations should be conducted by economic and not political principles (González, 2004). Although the Barco administration did not completely break with the tradition of alignment with the United States, it did create a new style in managing international politics. In a way, the close agreement with Washington on the issue of illicit drugs allowed the Colombian government to maintain differences in other areas of the binational agenda.

Concerning this, Tokatlian (1994), argues that political dissent did not alter the economic agreement with the US. Barco's government international activity, was built on three main components: the first one consisted of the foreign politics decentralization, seeking new areas in the Pacific, the second component was 
based on taking special care of the relationship with the United States and the third one, tried to foster a significant improvement in its connections with multilateral economic organizations through a conservative management of the economy, even though it substantially limited its financial autonomy. The Barco administration, believed that Colombia should prepare for the new international reality, therefore, the emphasis of its foreign politics had to be in the Pacific Rim. Alongside, Colombia remained within the Non-Aligned Movement (NAM), trying to maintain some autonomy from Washington (Cardona, 1990).

As proof of its relative international autonomy, Colombia maintained and expanded the contacts initiated during the previous government with Cuba, although the formal diplomatic contacts had been suspended since 1981, the meeting points between the two countries became frequent. As a result of the improvement in bilateral relations, Colombia and Cuba, signed on December 12 in the city of Barranquilla a partial scope agreement in which both countries expected to increase their trade by granting reciprocal tariff preferences. Callejas \& González (1998) indicate that the relationship with Cuba, not only occurred bilaterally, but also it was projected in multilateral scenarios, as it happened in 1989 in the United Nations Commission on Human Rights Framework, in which Colombia tried to avoid a political condemnation against the government of the island by abstaining and voting against the United States, because of the internal situation of Human Rights. One year later, both countries agreed on the United Nations Security Council, and along with Malaysia and Yemen, formed the UN's gang of four, which unified positions on the crisis in the Persian Gulf,
Namibia's independence, Arabs territories held by Israel and the U.S. invasion to Panama.

\section{Cesar Gaviria Trujillo (1990-1994).}

\section{A new international scenario}

with the same internal problems

President Gaviria's administration begins with the decline and end of the cold war as a backdrop. This new international scenario would open possibilities of rapprochement for historically distant states in diplomatic relations. Some of the most relevant purposes regarding international politics of this government are: the expansion of trade with various states, the internationalization of the Colombian economy and the pursuit of strengthening the bargaining power of the region. To Cardona \& Tokatlian (1991), President Gaviria, gave continuity to the international politics of the previous government, by retaining several former administration officials and maintaining the focus on economic issues, seeking greater openness to foreign trades, always defending law and international institutions. On the subject of internal conflict and peace, President Gaviria acknowledged the close connection between international politics and internal peace.

In this regard, Colombia along with Spain, Mexico and Venezuela formed, with the consent of the UN's Secretary General, and with the approval of the United States, the Support Group for the Peace Process in El Salvador, which led to the definitive peace agreement in 1992; and during the signing of the Framework Agreement between the Guatemalan National Revolutionary Unity (GNRU) and the government of Guatemala, the parties requested that Colom- 
bia, Spain, United States, Mexico and Venezuela formed the Group of Friends of the Guatemalan peace process. In addition, Colombia sought in the Organizations American States (OAS) the restitution of the power for the elected President of Haiti, Jean Bertrand Aristide, who was ousted in a coup in 1991 (Tokatlian, 1999). As for the relations with Cuba, which were well-established during the previous administration, the formal diplomatic relations between Colombia and the island were finally reactivated in October 1991. This fact had an impact on the public order situation and human rights in Colombia directly.

At the multilateral level, Colombia was linked to the Association of Caribbean States (ACS), in July 1994 and won the presidency of the group of NonAligned countries. At the end of its mandate, drug trafficking and human rights passed to the center of the international agenda, limiting its external maneuver as it needed the United States to contain and resolve the complex problem of drugs, which by that time threatened the institutions of the Colombian state seriously.

According to Garay, Angulo \& Chain (1994), in economic terms, Washington played an important role in achieving the Hercules credit worth US $\$ 1,775$ million for the period from 1991 to 1994 .

\section{Ernesto Samper (1994-1998). The narcotization of foreign relations}

President Ernesto Samper Pizano's liberal administration (1994-1998), began with serious obstacles in its relations with the United States, mainly because of the scandal that led to the confirmation of drug incomes (Cali Cartel) to his presidential campaign. Domestically, the continued deterioration of the human rights violations, made the international community perceive this situation as a sign of government weakness. In 1995, Samper took office of the NonAligned Movement. Which was presented as an opportunity to gain recognition and dynamism in his international activity, was also undervalued and squandered among other things, due to the lack of analysis and planning on the true scopes, costs and benefits of this international responsibility, the absence of an internal political consensus and the stigma that followed the movement from the time of its founding. According Socorro Ramirez, quoted by González (2004):

the country was involved in the greatest responsibility of its history without the decision being previously discussed by anyone and no sector of the society, not even the government itself, clearly knew the consequences, costs and benefits, of course, no premeditated plan or strategy to capitalize on behalf of the national interests (p. 278).

According to Rojas (2006), Samper's government crisis, showed the extent in which drug trafficking was installed in the Colombian society which sparked alarm in the United States about the real possibility of being in the presence of a state controlled by organized crime. The growing importance of Colombia in the global economy of cocaine and sustained increase in the links between armed groups with the drug economy were increasing the armed conflict, which led to the United States applied counternarcotics strategy thoroughly, affecting diverse populations by aerial spraying (Vargas, 1999). During the Samper administration, the United States sought to prevent Colombia from becoming a narco-democracy, reaching 
for the first time in over thirty years of armed conflict to publicly discredit a Colombian government. The fact of voiding the U.S. visa to President Samper, the disapproval in 1996 and 1997.

By the end of Samper's administration, the U.S. Department of Defense, said that the due to their ties to drug trafficking, the armed groups had reached high levels of sophistication and represented the biggest threat to the Colombian military. Since then the bilateral agenda while keeping the drug problem as the core, it began to be traversed most often by the armed conflict (Rojas, 2006).

\section{Andrés Pastrana (1994-1998). From Counternarcotics aid to international intervention in the Colombian armed conflict}

President Andres Pastrana's administration (1994-1998), began with a process of political negotiation with the FARC-EP guerrilla. The peace strategy of this government sought the total participation of the international community in the negotiation process, putting its emphasis on the United States. This situation became clear during the visit by President Pastrana to Washington in October 1998, at the meeting the two leaders agreed on the "Alliance against illicit drugs", this would be the basis of bilateral relations during this four-year term. Until then, the State Department said that it was possible to repeat in Colombia the strategic framework used in El Salvador in the late eighties. From this perspective the direct participation of troops is avoided and the sustained technical assistance is promoted through training, intelligence technology and specialized equipment with the aim of weakening the insurgency and force its decision to negotiate.
The support of the United States, to the peace negotiations, was affected as a result of the murder of three U.S. indigenous by the FARC-EP in March 1999. Washington's position regarding the FARC-EP became revealed through the visit of Thomas Pickerin in August 1999, who expressed his government's concern about the way he was conducting the "Demilitarized Zone" and the abuses that were occurring there. Since then, the United States conditioned its continued support to Colombia ${ }^{10}$, the design of a coherent plan for the fight against drug trafficking, which the Colombian government agreed unconditionally (Gonzalez, 2004).

Thus, the Plan Colombia that according to Rojas (2006), in 1998 had been presented by the Colombian president as a kind of Marshall Plan for economic and social development it rapidly became the basis of the anti-drug strategy of the United States for the entire Andean region. The Plan Colombia combined anti-drugs measures such as controlling chemical precursors, spraying illicit crops, destroying laboratories and seizure of cargo and air interdiction; and recovery strategies of state control over drug producing areas. Therefore, in its first phase, the plan is directed to the departments of Putumayo and Caquetá, major producers of cocaine and militarily controlled by the South Block of the FARC-EP. In the territorial deployment of the Plan, the military counternarcotics battalions were really important; they were trained and equipped by the United States. These, became the military component of drug policy. In practice, the Plan Colombia became the first step of direct US involvement in the

\footnotetext{
${ }^{10}$ In 1999, Colombia was the third country in the world to receive more military support from the United States.
} 
Colombian armed conflict, and despite handling cautiously Colombia's autonomy regarding the management of its internal conflict, there are issues of their own agenda in Colombia, which were affected by the armed conflict. Topics such as drug trafficking, democracy, human rights, and the lack of security guarantees for their investments were increasingly impacted by the escalation and degradation of war in Colombia (Rangel, 2001). There were not few criticisms aroused by the Plan Colombia and its implementation, for its militarist and interventionist nature, for example the European Parliament in its February 2001 statement, quoted by González (2004), expressed full support for the peace process and concerted solution to the conflict, recognizing the political and social dimensions.

In the same vein, Pinto (2001), referred to the implementation of Plan Colombia, from the point of view of some spokesmen of the Venezuelan government, which on several occasions had referred to that Plan Colombia saying it could provoke a military imbalance in the Andean region, particularly between Colombia and Venezuela. Military cooperation with the United States during Pastrana's government, in the words of former president "resulted in the upgrade of the existing aviation, avionics systems of the helicopters were improved in the army and a reconnaissance aircraft was equipped" (Vargas, 2010: 137).

\section{Álvaro Uribe Vélez (2002-2010). Securitization of Colombian foreign policy}

The first presidential victory of Alvaro Uribe (2002-2006), was marked by the following socio-political variables, vari- ables that contextualize the beginning of his administration: Termination by Pastrana's administration of the peace talks; a globalized international stage, with the United States as the dominant political and military hegemon, installing a new international agenda, focused on the global fight against terrorism. And the Andean Region was institutionally unstable and politically changing.

\section{The policy of Democratic Security}

The then Defence Minister Martha Lucia Ramirez, cited in the UNDP report (2003), defined the Defense Policy and Democratic Security (DSDP) as a longterm state policy to protect the population. The first strategy pursued by the State, was to contain and protect; Taking as a starting point the protection of vehicular free transit through the main roads of the country, providing security to travelers through military convoys escort, called "Vive Colombia y viaja por ella" which means "Live Colombia and travel through it" and the launch of the Meteor Plan. While territorial recovery control is started. According to Pizarro (2004), at the beginning of Uribe's administration, the National Police was absent from 160 municipalities, about $15 \%$ of all Colombian municipalities. In the second half of 2003 it was only the $5 \%$ of the municipalities the ones without state presence, and in 2004 there was permanent presence of the National Police in all municipalities of Colombia (Vargas, 2010).

At the international level the first step of Uribe's administration was placing the Colombian armed conflict in the "global crusade against terrorism" led by the United States after the attacks of September 11. In an international con- 
text marked by the positioning of terrorism as the main threat to the global security agenda, President Uribe, managed to present the Colombian armed conflict as a threat not only to the region since the FARC-EP, ELN and Autodefensas Unidas de Colombia (AUC), were considered terrorist organizations by the US State Department, and also involved with narcotics trafficking, the scope of their armed actions took on global dimensions. In this case, this relationship can be clearly seen between domestic policy and foreign policy of President Uribe. Relationship, in which longevity and degradation of the Colombian armed conflict, becomes relevant.

In this sense, according to Tokatlian (1999), each State, in whose territory a war is developed, directs part of its diplomacy to seek external support to solve its internal war. In Colombia, Uribe's administration diplomacy, sought internationally legitimate the utilization by the Colombian government, of a greater use of force, aimed at the political and military defeat of the insurgency and drug trafficking. Thus, the issue of security became the axis around which the foreign policy of President Uribe gravitated. From a theoretical approach, Uribe's government diplomacy was framed in the neo-realist school of international relations, since security became the central theme of his political actions. In this context, the safety as well as being the basis on which his government was built, it became the cornerstone of the process of decision making of its foreign policy, that is, the same (Jiménez, 2004) was securitized.

So that the assignment of the Colombian armed conflict in the global fight against terrorism, and his unconditional alliance with the United States, was successful because Colombia, maintained and consolidated military aid from the United States through Plan Colombia, thus ensuring the sustainability of the military component of Democratic Security. It was thus, as Plan Colombia went from being an anti-narcotics plan in their planning, to a counterterrorism plan in its name and counterinsurgency plan, in its execution, that is, the efforts of Uribe's administration, addressed to increase economic aid from the United States, to enhance the deterrent capability of the Armed Forces and put them in offensive attitude towards the insurgency.

\section{RESULTS}

Colombia's foreign policy has been profoundly marked by the intervention of the United States since the pronouncement of Marco Fidel Suarez from the early 20 s of last century, by expressing the Respice Polum.

In this regard, the South American country was the main beneficiary of the Alliance for Progress and concurrently Colombian military leadership was formed under the guidance of the Americans at Escuela de las Américas which certainly was decisive in the course of interaction with the rest of the system in the bipolar scenario.

With the collapse of the Cold War, Colombia, unlike most of the region, continued along the path of the "North Star", inviting it to participate as a relevant actor in the conflict through the implementation of Plan Colombia, thereby which, it advanced towards a securitization in the relationship with the other actors in the region, which led to a growing tension. 
In this same order of ideas, the period of greatest securitization, made a negative impact on the economic and trade relationship, denoting an obvious decline in exports towards traditional strategic partners such as Ecuador and Venezuela. It is demonstrated throughout the analysis that the need of the U.S. market and the banking loans, coupled with internal problems such as drug trafficking, subversion and terrorism have formed an almost structural dependence on North America which contrasts with the other countries in the region.

\section{CONCLUSION}

From the above analysis it can be said that while Colombia's foreign politics has sought universal diplomacy, relations with the United States have been the axis around which it has weighed its international activity, limiting or conditioning it in economical, political and in matters of internal security. Although this reliance has been continuous, there have been attempts to generate ruptures, but various factors have maintained over time such continuity, taking as reference the Respice Polum. In this process of dependency, you can find four different moments with a specific axis.

An initial moment founded in anticommunism (1948 - 1970), a second stage marked by the need for markets and loans (1974 - 1990), a third moment focused on the war on drugs (1990 - 2002) and fourth moment focused on the global fight against terrorism (2002-2010). The most direct consequences of this situation were: a. The noticeable distance between the region and mistrust of their neighbors, directly impacting migratory conditions of their nationals and an important sector of its exports;

b. The violation of the international law, as it happened in the attack on the camp of the guerrilla commander Raul Reyes in the Ecuadorian jungle;

c. The neglect and underestimation of important political and commercial spaces such as Europe and Latin America and the Caribbean, precisely the areas in which the international activity of the FARC-EP had its major developments. That is, the diplomacy of the FARC-EP did not have state counterweight. As it was stated by former President Alvaro Uribe Velez, in April 2009: "They had a great international diplomacy that exceeded the Colombian Foreign Ministry because they were not properly faced."

This situation has been aggravated among other things by the noticeable partiality that Colombian presidents have faced the country's foreign policy, which shows the absence of a state policy that seeks long-term international objectives. We have observed over the cold war our country has been oriented to assume as its own the United States policies, which is why the foreign policy has revolved around managing the political and economic agenda based on the White House strategic interests. Therefore we have been a strategic partner and also an unconditional ally of the capitalist world, even after the completion of the East-West conflict. 


\section{REFERENCES}

Atkins, G. (1991). América Latina en el sistema politico internacional. Buenos Aires: GEL.

Cardona, D. (1990). La administración Barco y los No Alineados, Revista Colombia Internacional, 9, 3-9.

Cardona, D. \& Tokatlian, J. (1991). Los desafíos de la política internacional Colombiana en los noventas, Revista Colombia Internacional, 14.

Cepeda, F. \& Pardo, R. (1989). La política exterior colombiana (1974-1986). Bogotá: Editorial Planeta.

Deas, M. (1988). El proceso de paz colombiano, 1982-1985 y sus implicaciones para Centroamérica, Documentos Ocasionales, 5.

Callejas \& González, R. (1998). Colombia y Cuba. Del distanciamiento a la cooperación. Barranquilla: Ediciones Uninorte.

Garay, L., Angulo, A. \& Cadena, C. (1994) Cultura de negociación: la experiencia de la deuda externa. Bogotá: CEREC-FESCOL.

González, R. (2004). "La política exterior de Colombia a finales del siglo XX. Primera aproximación", Investigación y Desarrollo, 12(2), 358-385.

Jiménez, N. (2004). "Implicaciones de la securitización en América desde la Particularidad colombiana". Revista Investigación y Desarrollo, 12(2), 244-257.

López, A. (1978). Testimonio Final. Bogotá: Imprenta Nacional.

Meléndez, J. (2014). Colombia y su participación en la guerra de Corea. Una reflexión tras 64 años de iniciado el conflicto, Revista Historia y memoria, 10(1), 199-239.

Pardo, R. (1987). La política exterior de la administración Barco. Análisis Político, 2, 3-9.

Pinto, M. (2001). "El Plan Colombia y los Procesos de Integración Subregional Andina. Visión desde Venezuela", Universidad Central de Venezuela, Presentation prepared for the XXIII Congreso Latín American Studies Association. Washington, D.C.

Pizarro, E. (2004). Una democracia asediada. Balance y perspectivas del conflicto armado en Colombia. Bogotá: Grupo Editorial Norma.

Rangel, A. (2001). Guerra contrainsurgente, conflictos armados en Malasia, Perú, Filipinas, El Salvador y Colombia. Bogotá: Intermedio Editores.

Rojas, D. (2006). Los Estados Unidos y la guerra en Colombia. Bogotá: Grupo Editorial Norma.

Rojas, D. (2010). La Alianza para el Progreso en Colombia. Retrieved from: http://www.ceecolombia.org/ckfinder/ userfiles/files/Ponencia\%20APP\%20 Congreso\%20RR I I\%2 0\%28Di ana\%20Rojas\%29.pdf

Tokatlian, J. (1994). "La política exterior del gobierno del presidente Virgilio Barco: En busca de la autonomía perdida" en: El gobierno de Barco. Política, economía y desarrollo social en Colombia, 1986-1990. Bogotá. Fedesarrollo - Fondo Cultural Cafetero.

Tokatlian, J. (1999). "Las diplomacias por la paz" Armar la paz es desarmar la guerra. Herramientas para lograr la paz. Bogotá: FESCOL, IEPRI, CEREC. 
Vargas, A. (2010). "Seguridad Democrática, conflicto interno armado y su regionalización” Seguridad en Democracia; Un reto a la violencia en América Latina. Buenos Aires: CLACSO, Colección Grupos de Trabajo.
Vargas, R. (1999). Drogas, máscaras y juegos. Narcotráfico y conflicto armado. Bogotá: Tercer Mundo Editores. 